\title{
Self-organization of ULF electromagnetic wave structures in the shear flow driven dissipative ionosphere
}

\author{
G. Aburjania ${ }^{1,2}$, K. Chargazia ${ }^{1,2}$, O. Kharshiladze ${ }^{2}$, and G. Zimbardo ${ }^{3}$ \\ ${ }^{1}$ I. Vekua Institute of Applied Mathematics, Tbilisi State University, 2 University str., \\ 0143 Tbilisi, Georgia \\ ${ }^{2}$ M. Nodia Institute of Geophysics, Tbilisi State University, 1 Aleqsidze str., \\ 0193 Tbilisi, Georgia \\ ${ }^{3}$ Physics Department, University of Calabria, Ponte P. Bucci, Cubo $31 \mathrm{C}, 87036$ Rende, Italy
}

Received: 18 July 2014 - Accepted: 28 July 2014 - Published: 26 August 2014

Correspondence to: K. Chargazia (khatuna.chargazia@gmail.com) and O. Kharshiladze (o.kharshiadze@mail.ru)

Published by Copernicus Publications on behalf of the European Geosciences Union \& the American Geophysical Union.

\section{Abstract}

This work is devoted to investigation of nonlinear dynamics of planetary electromagnetic (EM) ultra-low-frequency wave (ULFW) structures in the rotating dissipative ionosphere in the presence of inhomogeneous zonal wind (shear flow). Planetary EM

5 ULFW appears as a result of interaction of the ionospheric medium with the spatially inhomogeneous geomagnetic field. The shear flow driven wave perturbations effectively extract energy of the shear flow increasing own amplitude and energy. These perturbations undergo self organization in the form of the nonlinear solitary vortex structures due to nonlinear twisting of the perturbation's front. Depending on the features

10 of the velocity profiles of the shear flows the nonlinear vortex structures can be either monopole vortices, or dipole vortex, or vortex streets and vortex chains. From anaIytical calculation and plots we note that the formation of stationary nonlinear vortex structure requires some threshold value of translation velocity for both non-dissipation and dissipation complex ionospheric plasma. The space and time attenuation specifi15 cation of the vortices is studied. The characteristic time of vortex longevity in dissipative ionosphere is estimated. The long-lived vortices transfer the trapped medium particles, energy and heat. Thus they represent structural elements of turbulence in the ionosphere.

\section{Introduction}

${ }_{20}$ The interaction between the solar wind (SW) and terrestrial magnetosphere is the primary driver of many processes and phenomena occurring in the magnetosphere and consequently, in the ionosphere. However, many of the energy transfer processes have a sporadic/bursty character, and observations have highlighted that the vortexlike plasma flow structures are common in the Earth's magnetosphere. They prevail in the nightside plasma sheet (Hones, 1978, 1981, 1983; Keiling et al., 2009) and at the flank magnetospheric low-latitude boundary layers (LLBLs) (Fairfield et al., 2000; 
Otto and Fairfield, 2000; Hasegawa et al., 2004). Plasma vortex-like flows have also been observed on the middle to high-latitude boundary of the outer radiation belt by the Cluster spacecraft fleet (Zong et al., 2009). The plasma flow vortex found in the magnetotail is characterized by pronounced vortical motion in the plane that is approx-

5 imately parallel to the ecliptic plane. Vortex-like plasma flows in the plasma sheet are thought to be important in transportation of the kinetic energy from fast flow or bursty bulk flows (BBFs) in the magnetotail to the near Earth region (Snekvik et al., 2007; Keiling et al., 2009). A sudden braking and/or azimuthal deflection of BBFs may generate the plasma flow vortices at the boundary between the magnetotail plasma sheet and 10 the inner magnetosphere as suggested by Hasegawa (1979) and Vasyliunas (1984). Keika et al. (2009) have shown that plasma vortices are formed near the region where the earthward flows slow down and turn in azimuthal directions. The theoretical relation between the field-aligned current (FAC) and plasma vorticity showed that FACs are generated to transport transverse momentum along magnetic field lines (Pritchett 15 and Coroniti, 2000). Keiling et al. (2009) presented a scenario in which the plasma flow vortices in the plasma sheet generated the FAC of the substorm current wedge (SCW) at the beginning of the substorm expansion phase and coupled to the ionosphere, causing the ionospheric vortices.

In the present paper, we continue investigation of a special type of internal waves, 20 which appear in the ionosphere under the influence of the spatially inhomogeneous geomagnetic field and the Earth's rotation velocity (Aburjania et al., 2002, 2003, 2004, 2007). In the previous works generation mechanism and self-organization into the vortex structures is studied. Now, we are interested in large-scale (planetary) ultra-lowfrequency (ULF) electromagnetic (EM) vortex structures in the ionospheric medium 25 (consisting of electrons, ions and neutral particles), which have a horizontal linear scale $L_{\mathrm{h}}$ of order $10^{3} \mathrm{~km}$ and higher, a vertical scale $L_{\mathrm{v}}$ of altitude scale order $H\left(L_{\mathrm{v}} \approx H\right)$.

Observations (Gershman, 1974; Gossard and Hooke, 1975; Kamide and Chian, 2007) show also, that spatially inhomogeneous zonal winds (shear flows), produced by nonuniform heating of the atmospheric layers by solar radiation, permanently exist

$$
1433
$$

in the atmosphere and ionosphere layers. Herewith, investigation of the problem of generation and evolution of ionospheric EM ULF electromagnetic wave structures taking into account the inhomogeneous zonal wind (shear flow) becomes important.

\section{The governing equations}

5 We choose our model as a two-dimensional $\beta$-plane with sheared flow. Since the length of planetary waves $\left(\lambda \geq 10^{3} \mathrm{~km}\right)$ is comparable with the Earth's radius $R$, we investigate such notions in approximation of the $\beta$-plane, which was specially developed for analysis of large-scale processes (Pedlosky, 1978), in the "standard" coordinate system. In this system, the $x$ axis is directed along the parallel to the east,

10 the $y$ axis along the meridian to the north and the $z$ axis - vertically upwards (the local Cartesian system). For simplicity, the equilibrium velocity $\boldsymbol{V}_{0}$, geomagnetic field $H_{0}$, perturbed magnetic field $h$ and frequency of Earth's rotation $\Omega_{0}$ are given by formulas $\boldsymbol{V}_{0}=\boldsymbol{V}_{0}(y) e_{x}, \boldsymbol{H}_{0}\left(0,0,-H_{\mathrm{p}} \cos \theta\right), \boldsymbol{h}\left(0,0, h_{z}\right), \Omega_{0}\left(0,0, \Omega_{0} \cos \theta\right)$. Here and elsewhere $e\left(\boldsymbol{e}_{x}, \boldsymbol{e}_{y}, \boldsymbol{e}_{z}\right)$ denotes a unit vector, $H_{\mathrm{p}}=5 \times 10^{-5} \mathrm{~T}$ is the value of geomagnetic 15 field strength in the pole and we suppose that geomagnetic colatitude $\theta$ coincides with a geographical colatitude $\theta^{\prime}$. In the ionosphere the large-scale motions are quasihorizontal (two-dimensional) (Aburjania et al., 2002, 2003, 2006) and hydrodynamic velocity of the particles $V=\left(V_{x}, V_{y}, 0\right)$. The fluid is assumed to be incompressible and therefore a stream function $\boldsymbol{\psi}$ can be defined trough $\boldsymbol{V}=\left[\nabla \boldsymbol{\psi}, \boldsymbol{e}_{z}\right]$. Medium motion is 20 considered near the latitude $\varphi_{0}=\pi / 2-\theta_{0}$.

Not considering any more detail in the new under review branches of planetary waves (see Aburjania et al., 2002, 2003, 2004, 2007, 2011) we would like to note that beginning with the altitude of $80 \mathrm{~km}$ and higher, the upper atmosphere of the Earth is a strongly dissipative medium. Often when modelling large-scale processes for this region of the upper atmosphere, effective coefficient of Rayleigh friction between the ionospheric layers is introduced. The role of the ion friction rapidly increases at the altitudes above $120 \mathrm{~km}$ (Kelley, 1989; Kamide and Chian, 2007) and its analytical expression 
coincides with the Rayleigh friction formula (Aburjania and Chargazia, 2007). Therefore, often during a study of large-scale $\left(10^{3}-10^{4}\right) \mathrm{km}$, ULF $\left(10-10^{-6}\right) \mathrm{s}^{-1}$ wavy structures in the ionosphere, we will apply the well-known Rayleigh formula to dissipative force $F=-\Lambda V$, assuming the altitudes above $(80-130) \mathrm{km} \Lambda \approx 10^{-5} \mathrm{~s}^{-1}$ (Dickinson,

5 1969; Gosard and Hooke, 1975), and the altitudes above $130 \mathrm{~km} \wedge=N v_{\text {in }} / N_{\mathrm{n}}$, where $N$ and $N_{\mathrm{n}}$ denote concentrations of the charged particles and neutral particles, $v_{\text {in }}$ is frequency of collision of ions with molecules (Gershman, 1974; Kelley, 1989; Al'perovich and Fedorov, 2007).

The governing equations of the considered problem are the closed system of magne10 tohydrodynamic equations of the electrically conducting ionosphere (Gershman, 1974; Kelley, 1989; Aburjania et al., 2004, 2007; Al'perovich and Fedorov, 2007). The solution of the temporal evolution of inhomogeneously sheared flow reduces to solution of the set of nonlinear partial differential equations for $\psi$ and magnetic field perturbation, $h_{z}$ (see Aburjania et al., 2002):

$$
\begin{aligned}
{ }_{15} & \left(\frac{\partial}{\partial t}+V_{0}(y) \frac{\partial}{\partial x}\right) \Delta \psi+\left(\beta-V_{0}^{\prime \prime}\right) \frac{\partial \psi}{\partial x}+C_{\mathrm{H}} \frac{\partial h}{\partial x}+\Lambda \Delta \psi=J(\psi, \Delta \psi), \\
& \left(\frac{\partial}{\partial t}+V_{0}(y) \frac{\partial}{\partial x}\right) h-\beta_{\mathrm{H}} \frac{\partial \psi}{\partial x}+\delta \cdot C_{\mathrm{H}} \frac{\partial h}{\partial x}=J(\psi, h) .
\end{aligned}
$$

\section{Here}

$$
\begin{aligned}
\beta & =\frac{\partial 2 \Omega_{0}}{\partial y}=-\frac{1}{R} \frac{\partial}{\partial \theta}\left(2 \Omega_{0}\right)=\frac{2 \Omega_{0} \sin \theta_{0}}{R}, \\
20 \quad \beta_{\mathrm{H}} & =\frac{e N}{\rho c} \frac{\partial H_{0 z}}{\partial y}=-\frac{N}{N_{\mathrm{n}}} \frac{e H_{\mathrm{p}}}{M R c} \sin \theta_{0}<0, \quad V_{0}^{\prime \prime}(y)=\frac{d^{2} V_{0}(y)}{d^{2} y}, \\
h & =\frac{e N}{N_{\mathrm{n}} M c} h_{z}, \quad C_{\mathrm{H}}=\frac{c}{4 \pi e N} \frac{\partial H_{0 z}}{\partial y}=-\frac{c H_{\mathrm{p}}}{4 \pi e N R} \sin \theta_{0}<0, \\
\Delta & =\frac{\partial^{2}}{\partial x^{2}}+\frac{\partial^{2}}{\partial y^{2}}, \quad J(a, b)=\frac{\partial a}{\partial x} \cdot \frac{\partial b}{\partial y}-\frac{\partial a}{\partial y} \cdot \frac{\partial b}{\partial x}
\end{aligned}
$$

$\rho=N_{\mathrm{n}} M$ is density of neutral particles; $m$ and $M$ are masses of electrons and ions (molecules); $e$ is the magnitude of the electron charge; $c$ is the light speed. Further we consider a motion in neighborhood of fixed latitude $\left(\theta=\theta_{0}\right)$. The dimensionless pa5 rameter $\delta$ is introduced here for convenience. In the ionospheric $E$ region $(80-150) \mathrm{km}$, where the Hall effect plays an important role, this parameter is equal to unity $(\delta=1)$. In the $F$ region $(200-600) \mathrm{km}$, where the Hall effect is absent, $\delta$ turns to zero $(\delta=0)$.

The system of Eqs. (1) and (2), at corresponding initial and boundary conditions, describes nonlinear evolution of the spatial two-dimensional large-scale ULF electro10 magnetic perturbations in sheared incompressible ionospheric $E$ and $F$ regions.

From the Eqs. (1) and (2) we determine the temporal evolution of the energy of wavy structures, $E(x, y, t)$

$$
\frac{\partial E}{\partial t}=\int V_{0}^{\prime}(y) \frac{\partial \psi}{\partial x} \frac{\partial \psi}{\partial y} \mathrm{~d} x \mathrm{~d} y-\Lambda \int|\nabla \psi|^{2} \mathrm{~d} x \mathrm{~d} y
$$

We note that in the absence of zonal flow $\left(V_{0}=0\right)$ and Rayleigh friction $(\Lambda=0)$ the wavy structure energy is conserved.

Therefore, the existence of sheared zonal flow can be considered as the presence of an external energy source. One can see, that it (term with $V_{0}(y)$ in Eq. 4) feeds the medium with external source of energy for generation of the wave structures (development of the shear flow instability). The shear flows can become unstable transiently until the condition of the strong relationship between the shear flows and wave perturbations is satisfied (Chagelishvili et al., 1996; Aburjania et al., 2006), e. i. the perturbation falls into amplification region in the wave number space. Leaving this region, e. i. when the perturbation passes to the damping region in the wave vector space, 25 it returns an energy to the shear flow and so on (if the nonlinear processes and selforganization of the vortex structure will not develop before) (Aburjania et al., 2006). The experimental and observation data shows the same (Gossard and Hooke, 1975; Pedlosky, 1987; Gill, 1982). 


\section{Criterion of instability of ULF PEMW wave structures in the ionopashere with the shear flow}

The character of the shear flow much defines the evolution of the wave perturbation in the medium. Therefore, the shear flow in the hydrodynamics and in magnetohydrody-

5 namics are often unstable (Gossard and Hooke, 1975; Mikhailovskii, 1974; Timofeev, 2000). Existing of the term proportional to $V_{0}^{\prime \prime}=d^{2} V_{0} / \mathrm{d} y^{2}$ in Eq. (1) is related with the criterion (condition) of instability of the shear flow. In the linear approximation for the small scale perturbations of the form $\psi(x, y, t), h(x, y, t)=\left(\psi_{1}(y), h_{1}(y)\right) \exp \left(i k_{x} x-i \omega\right)$ from the Eqs. (1) and (2) follows the equation of Ohr-Zomerfeld

$$
\begin{aligned}
10 i \frac{\Lambda}{\omega-k_{x} V_{0}}\left(\frac{d^{2}}{d y^{2}}-k_{x}^{2}\right) \psi_{1} & +\left(\frac{d^{2}}{d y^{2}}-k_{x}^{2}\right) \psi_{1}-\frac{k_{x}\left(\beta-V_{0}^{\prime \prime}\right)}{\omega-k_{x} V_{0}} \psi_{1} \\
& +\frac{k_{x}^{2} C_{\mathrm{H}} \beta_{\mathrm{H}}}{\left(\omega-k_{x} V_{0}\right)\left[\omega-k_{x}\left(C_{\mathrm{H}}+V_{0}\right)\right]} \psi_{1}=0 .
\end{aligned}
$$

Neglecting the dissipation terms $(\Lambda \rightarrow 0)$ from Eq. (5) we get

$$
\psi_{1}^{\prime \prime}-k_{x}^{2} \psi_{1}-\frac{k_{x}\left(\beta-V_{0}^{\prime \prime}\right)}{\omega-k_{x} V_{0}} \psi_{1}+\frac{k_{x}^{2} C_{\mathrm{H}} \beta_{\mathrm{H}}}{\left(\omega-k_{x} V_{0}\right)\left[\omega-k_{x}\left(C_{\mathrm{H}}+V_{0}\right)\right]} \psi_{1}=0
$$

where $\psi_{1}^{\prime \prime}=d^{2} \psi_{1} / \mathrm{d} y^{2}$. Equation (6) represents modification of known Rayleigh equation (Timofeev, 2000) (at $\beta_{\mathrm{H}} \rightarrow 0$ ). For determination shear flow instability criterion in our case Eq. (6) should be multiplied by $\psi_{1}^{*}$, excluding complex-conjugate expression and integrating obtained one between the edges $y_{1}$ and $y_{2}$ of the plasma flux:

$20 \int_{y_{1}}^{y_{2}} \frac{d}{\mathrm{~d} y}\left(\psi_{1}^{*} \frac{d \psi_{1}}{\mathrm{~d} y}-\psi_{1} \frac{d \psi_{1}^{*}}{\mathrm{~d} y}\right) \mathrm{d} y-\int_{y_{1}}^{y_{2}}\left[\frac{1}{\omega-k_{x} V_{0}}-\frac{1}{\omega^{*}-k_{x} V_{0}}\right] k_{x}\left(\beta-V_{0}^{\prime \prime}\right)\left|\psi_{1}\right|^{2} \mathrm{~d} y-$ 1437

$$
\int_{y_{1}}^{y_{2}}\left[\frac{k_{x}^{2} C_{\mathrm{H}} \beta_{\mathrm{H}}}{\left(\omega-k_{x} V_{0}\right)\left[\omega-k_{x}\left(C_{\mathrm{H}}+V_{0}\right)\right]}-\frac{k_{x}^{2} C_{\mathrm{H}} \beta_{\mathrm{H}}}{\left(\omega^{*}-k_{x} V_{0}\right)\left[\omega^{*}-k_{x}\left(C_{\mathrm{H}}+V_{0}\right)\right]}\right]\left|\psi_{1}\right|^{2} \mathrm{~d} y=0 .
$$

Supposing, the frequency of the perturbation $\omega=\omega_{0}+i \gamma$ to be complex, and wave vector $k_{x}$ - the real, imaginary part of the Eq. (7) can be written as:

$52 \gamma \int_{y_{1}}^{y_{2}} \frac{k_{x}}{\left(\omega_{1}^{2}+\gamma^{2}\right)}\left[\beta-V_{0}^{\prime \prime}-\frac{k_{x} C_{\mathrm{H}} \beta_{\mathrm{H}}}{\omega_{2}^{2}+\gamma^{2}} \omega_{3}\right]\left|\psi_{1}\right|^{2} \mathrm{~d} y=0$

where $\omega_{1}=\omega_{0}-k_{x} V_{0}, \omega_{2}=\omega_{0}-k_{x}\left(C_{\mathrm{H}}+V_{0}\right), \omega_{3}=2 \omega_{0}-k_{x}\left(C_{\mathrm{H}}+V_{0}\right)$. In case of $\omega_{1}, \omega_{2}, \omega_{3}, \gamma,\left|\Psi_{1}^{2}\right|>0$, from Eq. (8) follows the condition of the linear instability of the shear flow:

$10 \beta-V_{0}^{\prime \prime}-\frac{k_{x} C_{\mathrm{H}} \beta_{\mathrm{H}}}{\omega_{2}^{2}+\gamma^{2}} \omega_{3}=0$.

When $\omega_{0}^{\mathrm{f}}=k_{x}\left(C_{\mathrm{H}}+V_{0}\right), \quad C_{\mathrm{H}} \gg V_{0}$ and correspondingly, $\omega_{2} \approx 0, \omega_{3} \approx k_{x} C_{\mathrm{H}}$, from Eq. (9) we have:

$\beta+\frac{k_{x}^{2} C_{\mathrm{H}}^{2}\left|\beta_{\mathrm{H}}\right|}{\gamma^{2}}-V_{0}^{\prime \prime}=0$.

For the critical (resonance) level of the ionosphere, where the phase velocity of the slow waves $V_{\mathrm{ph}}=\omega^{\mathrm{s}} / k_{x}$ can coincide with the winds speed, $V_{\mathrm{ph}}=V_{0}\left(y_{r}\right)$ (i.e. $\omega_{1}=\omega_{0}-$ $k_{x} v_{0} \approx 0, \omega_{2} \approx-k_{x} C_{\mathrm{H}}, \omega_{3} \approx-k_{x} C_{\mathrm{H}},\left|\omega_{2}\right| \gg \gamma$ ), equality (9) can be rewritten as:

${ }_{20} \bar{\beta}-V_{0}^{\prime \prime}(y)=0$. 
Conditions (9)-(11) can be called as modified Rayleigh instability condition $\left(V_{0}^{\prime \prime}=0\right)$ for ULF PEMW at corresponding parameters of the zonal flow, wave and medium. This condition (10) (or 11) for some resonance point $y=y_{r}$ of the shear flow is the necessary condition for the shear flow instability.

5 For the description of the features of unstable oscillations fulfilling the resonance condition with the shear flow $V_{\text {ph }}=V_{0}\left(y_{r}\right)$ and Eq. (9), then Eq. (6) can be written in the following form:

$\psi_{1}^{\prime \prime}-k_{x}^{2} \psi_{1}+\frac{V_{0}^{\prime \prime}(y)-\bar{\beta}}{V_{0}\left(y_{r}\right)-V_{0}(y)} \psi_{1}=0$.

10 According to oscillatory theory (Timofeev, 2000), finite conditions (12) can have the discrete number of eigen functions $\psi_{1}^{(n)}$ with corresponding eigen values $k^{(n)}$ and frequencies $\omega^{(n)}=k^{(n)} V_{0}\left(y_{r}\right)$ if only potential $U_{r}(y)$ fulfills the condition

$U_{r}(y)=\frac{V_{0}^{\prime \prime}(y)-\bar{\beta}}{V_{0}\left(y_{r}\right)-V_{0}(y)}>0$.

15 Concrete condition for existence of the eigen functions $\psi_{1}^{(n)}$ depends on the concrete type of the shear flow velocity profile and correspondingly, on the type of $U_{r}(y)$. Criterion (13) presents necessary condition of the shear flow instability.

It must be mentioned, that for the waves fulfilling the necessary conditions of instability and resonance $V_{\mathrm{ph}}=V_{0}\left(y_{r}\right)$, both - dividend and divisor in Eq. (13) become zero 20 at $y=y_{r}$. Solving this uncertainty by means of L'Hopital's rule, we get enough condition of the shear flow instability:

$U_{r}\left(y_{r}\right)=\left.\frac{V_{0}^{\prime \prime}(y)-\bar{\beta}}{V_{0}\left(y_{r}\right)-V_{0}(y)}\right|_{y=y_{r}}=-\frac{V_{0}^{\prime \prime \prime}\left(y_{r}\right)}{V_{0}^{\prime}\left(y_{r}\right)}>0$.

In the earth atmosphere $\beta+k_{x}^{2} C_{\mathrm{H}}^{2}\left|\beta_{\mathrm{H}}\right| / \gamma^{2}$ and $\bar{\beta}$ can be greater or less than $V_{0}^{\prime \prime}$. Thus, in the ionosphere episodic generation of such zonal wind is possible, that at some critical layer $y=y_{r}$ the conditions (9)-(14) can be fulfilled, which is the reason of instability during some time, after which the zonal wind reorganizes and becomes 5 again stabile.

\section{Shear flow driven nonlinear solitary vortex structures}

As it is shown in the previous section, spontaneously generated ULF PEMW waves at different layers of the ionosphere at definite moment of evolution become unstable (at fulfilling instability condition 13 and 14) intensively pump shear flow energy (particu-

10 larly, during $0<\tau \leq \tau^{*}$ ). Getting energy, amplitudes of PEMW grow up (several times) and correspondingly, the nonlinear processes come into play. Herewith, in initial dynamic Eqs. (1)-(5) nonlinear terms become sufficient and the whole system should be analyzed.

Let us tern to study of the influence of the nonlinear effects on the dynamics of 15 ULF PEWM studied theoretically by us in the previous works (Aburjania et al., 2002, $2003,2004,2007)$ in non-dissipative ionosphere. The results of observations and missions show (Cmyrev et al., 1991) as it was described above, that the nonlinear solitary vortex structures can be generated at different layers of the atmosphere-ionospheremagnetosphere. These structures transfer the trapped particles of medium. Therefore, 20 relation of rotational velocity of particles $U_{C}$ with the motion velocity of the nonlinear structures $U$ is defined by $U_{C} / U \geq 1$ (Kamenkovich and Monin, 1978),

Let us introduce temperature $T$ and spatial $L$ characteristic scales of nonlinear structures. By virtue of Eq. (1) we can get the following relation between the values: $U_{C} \sim V, U \sim L / T$. Analogously, for the nonlinear and inertial terms we have:

$25(V \nabla) V /(\partial V / \partial t) \sim V /(L / T) \sim U_{C} / U \geq 1$. Thus, nonlinearity plays an important role for the wave processes, fulfilling $U_{\mathrm{C}} \geq U$. This estimation shows, that the nonlinear terms play an important role in the dynamics of ULF PEMW linear evolution of which is well 
described in our previous works (Aburjania et al., 2002, 2003, 2004, 2007). Inequality $U_{C} \geq U$ coincides with anti-twisting condition, only after fulfilling which the initial dynamic Eqs. (1) and (2) have solitary vortex solution (Williams and Yamagata, 1984).

From general theory of the nonlinear waves it is known (Whitham, 1974), that if in

5 the system the nonlinearity is sufficient then superposition is not applicable in this case and plane wave solution is not correct. Nonlinearity distorts wave profile and then it becomes different from sinusoid. If in nonlinear system dispersion is absent, all small amplitude waves with different wave numbers $k$ spread with same velocities and have possibility to interact with each other for a long time. Thus, even small nonlinearity 10 leads to storing of distortion. Such nonlinear distortions, as a rule, lead to growth of the wave front twisting and its breaking or bow shock formation. At the dispersion, he phase velocities o the waves with different $k$ are not equal, they spread with different velocities and do not interact with each other. Therefore, the wave packet have tendency to sprawl down and at small amplitude dispersion can compete with nonlinearity.

15 In consequence, the wave even before its breaking can split into separate wave packets and the bow shock will not be formed. Actually, in the real atmosphere the bow shock will not be formed arbitrarily (Shakina, 1985). First, this means that in atmosphereionosphere media dispersion is strongly pronounced and sufficiently competes with nonlinear distortions. If wave front nonlinear twisting will be compensated by disper20 sion, then stationary waves can be formed - solitary vortices, propagating in medium without changing its shape.

It must be mentioned also, that the satellite and ground based observations clearly indicate an existence of the zonal winds (flows) at different layers of the ionosphere (Gershman, 1974). At interaction with zonal flows the wave perturbation obtains an additional dispersion and new source of amplification and the nonlinearity affects their dynamics. Thus ionospheric medium with shear flows creates itself favorable conditions for the formation of the nonlinear stationary vortex structures.

Thus, our goal is to find stationary solution of Eqs. (1) ad (2) (in non-dissipative case $\Lambda=0) \bar{\psi}=\psi(\eta, y)$ and $h=h(\eta, y)$ spreading along the parallels (along $x$ axis) with

constant velocity $U=$ const without changing the form, where $\eta=x-U \tau$. We consider the case when the localized (even due to one coordinate) structure propagates on the background of mean zonal wind with non-uniform velocity $V_{0}(y)$.

Transforming the coordinates and taking into account that $\partial / \partial \tau=-U \partial / \partial \eta$, the sys5 tem (1) and (2) can be written as:

$$
\begin{aligned}
& -U \frac{\partial}{\partial \eta} \Delta \Psi+\beta \frac{\partial \Psi}{\partial \eta}+C_{\mathrm{H}} \frac{\partial h}{\partial \eta}-J(\Psi, \Delta \Psi)=0, \\
& \left(C_{\mathrm{H}}-U\right) \frac{\partial h}{\partial \eta}-\beta_{\mathrm{H}} \frac{\partial \Psi}{\partial \eta}-J(\Psi, h)=0 .
\end{aligned}
$$

Here, the stream function is introduced:

${ }_{10} \Psi(\eta, y)=\Phi_{0}(y)+\bar{\psi}(x, y)$.

And the velocity potential $\Phi_{0}(y)$ of the shear flow due to the notations:

$V_{0}(y)=-\frac{d \Phi_{0}(y)}{\mathrm{d} y}$.

15 Solution of Eq. (16) can be given as:

$h(\eta, y)=\frac{\beta_{\mathrm{H}}}{C_{\mathrm{H}}-U} \Psi$,

Further, substituting Eq. (19) into Eq. (15) and with analogous transformation we get Jacobean:

20 $J\left(\Delta \Psi-\left(\beta+\frac{C_{\mathrm{H}} \beta_{\mathrm{H}}}{C_{\mathrm{H}}-U}\right) y, \Psi-U y\right)=0$.

General solution of Eq. (20) has a form (Aburjania, 2006):

$\Delta \Psi-\frac{C_{\mathrm{H}} \bar{\beta}-U \beta}{C_{\mathrm{H}}-U} y=F(\Psi-U y)$, 
where $F(\xi)$ - an arbitrary function of its argument.

We will investigate bellow the solitary vortex structures in the ionosphere for different profiles of the shear flow velocity (Eq. 18).

\section{4.1 The monopole vortex solutions}

Let us consider the case, when the shear flow velocity is given by harmonic function

$\Phi_{0}(y)=a_{0} \sin \left(æ_{0} y\right)$,

where $a_{0}$ characterizes amplitude, $æ_{0}$ - transversal scale of the shear flow.

In this case, choosing the linear $F$ function:

$F=\kappa^{2}(\Psi-U y)$,

where $\kappa^{2}=\left(C_{\mathrm{H}} \bar{\beta}-U \beta\right) /\left[U\left(C_{\mathrm{H}}-U\right)\right]=$ const $>0$, Eq. (21) can be transformed into:

15

$\Delta \Psi-\kappa^{2} \Psi=\left(\kappa^{2}+æ_{0}^{2}\right) \Phi_{0}$

solution of which obtains the form

$\Psi=b_{1} K_{0}(k r)-\Phi_{0}(y)$

where $K_{0}$ - Mcdonalds function of Oth order; $r=\left(\eta^{2}+y^{2}\right)^{1 / 2}$ and $b_{1}=$ const - ampli20 tude of structure.

On the basis of solution (25) the medium velocity components $V_{x}$ and $V_{z}$ can be determined:

$V_{x}=\frac{b_{1} k}{r} \cdot y \cdot K_{1}(k r)+æ_{0} a_{0} \cos \left(æ_{0} y\right), \quad V_{y}=-\frac{b_{1} k}{r} \cdot \eta \cdot K_{1}(k r)$.

Parameter $b_{1}$ can be estimated from the boundary conditions. Thus, solution (26) depends on three parameters: from structure velocity $U$, characteristic transversal scale $æ_{0}$ and amplitude of the shear flow. The solution is strongly localized, being solitary monopole on the background of zonal harmonic wind, which behaves asymptotically $\sim \exp (-\kappa r) / \sqrt{r}$ at $r \rightarrow \infty$.

\subsection{The stationary vortex streets in the nondissipative ionosphere}

Vortex streets of various shapes can be generated in conventional liquid and plasma media with a sheared flow as a result of the nonlinear saturation of the KelvinHelmholtz instability (Gossard and Hooke, 1975; Kamide and Chian, 2007).

Thus, we will seek the solution of the nonlinear dynamic Eqs. (1) and (2) (in nondissipative stage, when $\Lambda \approx 0)$ in the form $\psi=\psi_{0}(\eta, y), h=h(\eta, y)$, where $\eta=x-U \tau$, i.e. the stationary solitary structures, propagating along $x$ axis (along the parallels) with velocity $U=$ const without changing its' shape. In accordance to Aburjania and Chargazia (2007), system of Eq. (1), (2) has the solution

${ }_{15} h(\eta, y)=\frac{\beta_{\mathrm{H}}}{C_{\mathrm{H}}-U} \Psi$,

$\Delta \psi_{0}-v_{0}^{\prime}(y)-\frac{C_{\mathrm{H}} \beta^{\prime}-U \beta}{C_{\mathrm{H}}-U} y=F\left(\psi_{0}-\int^{y} v_{0}(y) \mathrm{d} y-U y\right)$,

with $F(\xi)$ being an arbitrary function of its argument and $\Delta=\partial^{2} / \partial \eta^{2}+\partial^{2} / \partial y^{2}$. Vortex streets have complicated topology and can occur when the function $F(\xi)$ in Eq. (28) is nonlinear (Petviashvili and Pokhotelov, 1992; Aburjania, 2006).

In Eq. (28) we assume that a nonlinear structure propagates with the velocity $U$ satisfying the condition

$U=\frac{\beta^{\prime}}{\beta} C_{\mathrm{H}} \cdot$ 
For this case, choosing $F$ to be a nonlinear function, $F(\xi)=\psi_{0}^{0} \kappa^{2}\left(\exp \left(-2 \xi / \psi_{0}^{0}\right)\right.$ (Petviashvili and Pokhotelov, 1992; Aburjania, 2006), we can reduce Eq. (28) to

$\Delta\left(\psi_{0}-U y\right)=\psi_{0}^{0} k^{2} \exp \left[-2\left(\psi_{0}-U y\right) / \psi_{0}^{0}\right]$.

Then we introduce the new stream function

$\Psi_{0}(\eta, y)=\Phi_{0}(y)+\psi_{0}(x, y)$

and the velocity potential $\Phi_{0}(y)$ of the background sheared zonal flow,

${ }_{10} V_{0}(y)=\frac{d \Phi_{0}(y)}{\mathrm{d} y}$

The stream function of the background sheared flow $\Phi_{0}(y)$ can be chosen to have the form

$\Phi_{0}(y)=U y+\psi_{0}^{0} \ln \left(æ_{0} y\right)$.

Here, $\psi_{0}^{0}$ is the amplitude of the vortex structure, $2 \pi / k$ kappa is its characteristic size, and $2 \pi / æ_{0}$ is the nonuniformity parameter of the background sheared flow.

Taking into account Eq. (31) and using stream function (33), we can write vortex Eq. (30) as

$\Delta \psi_{0}=\psi_{0}^{0} æ_{0}^{2}\left[\frac{\kappa^{2}}{æ_{0}^{2}} e^{-2 \psi_{0} / \psi_{0}^{0}}-1\right]$.

This equation has the solution (Mallier and Maslowe, 1993)

$\psi_{0}(\eta, y)=\psi_{0}^{0} \ln \left[\frac{c h(k y)+\sqrt{1-æ_{0}^{2}} \cos (\kappa \eta)}{c h\left(æ_{0} y\right)}\right]$,

which describes a street of oppositely circulating vortices. Substituting solution (35) and stream function (33) into Eq. (31), we arrive at the final solution

${ }_{5} \Psi_{0}(\eta, y)=U y+\psi_{0}^{0} \ln \left[\operatorname{ch}(\kappa y)+\sqrt{1-æ_{0}^{2}} \cos (\kappa \eta)\right]$.

Equations (35), (33), and (32) yield the following expressions for the velocity components of the medium and sheared flow:

$$
\begin{aligned}
& V_{x}(\eta, y)=U+\psi_{0}^{0} k \frac{\operatorname{sh}(k y)}{c h(k y)+\sqrt{1-æ_{0}^{2}} \cos (k \eta)}, \\
& V_{y}(\eta, y)=\psi_{0}^{0} k \frac{\sqrt{1-æ_{0}^{2}} \sin (k \eta)}{c h(k y)+\sqrt{1-æ_{0}^{2}} \cos (k \eta)}, \\
& { }_{10} \quad V_{0}(y)=U+\psi_{0}^{0} æ_{0} \operatorname{th}\left(æ_{0} y\right) .
\end{aligned}
$$

For $æ_{0}=1$, solution (37) describes a background flow of the type of sheared zonal flow with velocity (Eq. 39). For $æ_{0}^{2}<1$, a street of cyclonic-type vortices forms in the middle of the zonal flow with velocity (Eq. 39) (Fig. 1). A solution like that described by

15 Eq. (37) and (38), with closed current lines in the form of cat's eyes, was for the first time obtained by Kelvin.

The vortex structures move with velocity (Eq. 29). If we take into account that $\beta_{\mathrm{H}}<0, \beta^{\prime}=\beta-\left|\beta_{\mathrm{H}}\right|<0$ as far as $\left|\beta_{\mathrm{H}}\right|>\beta>0, C_{\mathrm{H}}<0$ from expression Eq. (29) follows $U>0$. For $E$ region the characteristic parameters $N / N_{\mathrm{n}}=5 \times 10^{-7}, \Omega_{\mathrm{H}}=e H_{\mathrm{p}} /(M c) \sim$ ${ }_{20} 10^{3} \mathrm{~s}^{-1}, R=6.4 \times 10^{6} \mathrm{~m}, 2 \Omega_{0} \cong 10^{-4} \mathrm{rad} \mathrm{s}^{-1}$, we get that:

$$
\begin{aligned}
& \beta=2 \Omega_{0} \sin \theta_{0} / R \sim 0.8 \times 10^{-11} \mathrm{~m}^{-1} \mathrm{~s}^{-1}\left|C_{\mathrm{H}}\right| \approx 10 \mathrm{~km} \mathrm{~s}^{-1}, \\
& \left|\beta_{\mathrm{H}}\right|=\left(N /\left(N_{\mathrm{n}} R\right)\right) \Omega_{\mathrm{H}} \sin \theta_{0} \approx 4 \times 10^{-11} \mathrm{~m}^{-1} \mathrm{~s}^{-1} .
\end{aligned}
$$


Thus, the vortices move with velocity $U \approx 4\left|C_{\mathrm{H}}\right|>\left|C_{\mathrm{H}}\right|$ along the parallels to the east. Therefore, this velocity is greater than the phase one of the corresponding linear periodic waves $U>\left|C_{\mathrm{H}}\right| \approx 10 \mathrm{~km} \mathrm{~s}^{-1}$. So, the vortices don't come into resonance with the linear waves and don't loose energy on their excitation (Stepanyants and Fabrikant, 5 1992).

For estimation of the linear scale of the vortex structures let's remember the general formal relation between the dispersion equation of the linear waves and with so-called modified dispersion equation of the nonlinear structures (Petviashvili and Pokhotelov, 1992; Aburjania, 2006). This is coupling of the phase velocity of linear wave $V_{p}=\omega / k$ with motion velocity of the nonlinear structures $U:-\omega / k \rightarrow U$; relation of the wave vector $k$ of the linear disturbances with the characteristic linear scale of the vortex $d:-k \rightarrow d^{-1}$. Taking into account this fact for characteristic scale of the fast vortex structures we get:

$d_{15}^{f}=\left(\frac{\left|C_{\mathrm{H}}\right|}{\beta}\right)^{1 / 2}$.

And for the slow Rossby type vortex structures from the initial equations we get:

$d^{S}=\left(\frac{U}{\beta}\right)^{1 / 2}$

Substituting in these expressions the typical for the Earth's ionosphere numerical 20 values $\left|C_{\mathrm{H}}\right| \approx 10 \mathrm{~km} \mathrm{~s}^{-1}, \beta \approx 10^{-11} \mathrm{~m}^{-1} \mathrm{~s}^{-1}$, we find for fast structures $d^{\mathrm{f}} \approx 10^{4} \mathrm{~km}$. For slow Rossby-type vortices $U \approx 10 \mathrm{~m} \mathrm{~s}^{-1}$ and we can obtain $d^{\mathrm{s}} \approx 10^{3} \mathrm{~km}$.

For magnetic field perturbation from Eqs. (27) and (36), we can obtain the following estimation:

${ }_{25}|h| \approx\left|\beta_{\mathrm{H}}\right| \cdot d$,

valid for both the fast and slow modes. For the ionospheric conditions $\left|\beta_{H}\right| \approx 4 \times$ $10^{-11} \mathrm{~m}^{-1} \mathrm{~s}^{-1}$, thus using the estimations to carried out above, we may conclude that fast vortical motion generate magnetic pulsations $h^{\dagger} \approx 10^{-4} T$, while in case slow Rossby-type vortical motions $-h^{\mathrm{s}} \approx 10^{-5} T$.

Note that nonlinear stationary Eq. (28) also has an analytic solution in the form of a Larichev-Reznik cyclone-anticyclone dipole pair and other class of solitary solutions by different profiles of background shear flows (Petviashvili and Pokhotelov, 1992; Jovanovich et al., 2002; Aburjania, 2006; Aburjania et al., 2003, 2004, 2007).

\section{Attenuation of the vortex streets in the dissipative ionosphere}

10 In the dissipative approximation $(\Lambda \neq 0)$, we switch to the above self-similar variables $(\eta$ and $y$ ) and take into account the relationship $\partial / \partial \tau=-U \partial / \partial \eta$, which then holds. As a result, we can write Eqs. (1) and (2) as

$$
\begin{aligned}
& -U \frac{\partial}{\partial \eta} \Delta \Psi+\beta \frac{\partial \Psi}{\partial \eta}+C_{\mathrm{H}} \frac{\partial h}{\partial \eta}+\Lambda \Delta \psi-J(\Psi, \Delta \Psi)=0 \\
& \left(C_{\mathrm{H}}-U\right) \frac{\partial h}{\partial \eta}-\beta_{\mathrm{H}} \frac{\partial \Psi}{\partial \eta}-J(\Psi, h)=0 .
\end{aligned}
$$

Equation (44) has the solution

$$
h(\eta, y)=\frac{\beta_{\mathrm{H}}}{C_{\mathrm{H}}-U} \Psi \text {. }
$$

Substituting solution (45) into Eq. (43), take into account the expression (29) and

$$
\left(D_{\eta}+\frac{\Lambda}{U}\right) \Delta \Psi=0
$$


where

$D_{\eta}=\frac{\partial}{\partial \eta}+\frac{1}{U}\left(\frac{\partial \Psi}{\partial \eta} \frac{\partial}{\partial y}-\frac{\partial \Psi}{\partial y} \frac{\partial}{\partial \eta}\right)$

The Eq. (46) yield a solution as

${ }_{5} \quad \Psi=\Psi_{0} \cdot \exp \left(-\frac{\Lambda}{U} \eta\right)$

Here the zero ${ }^{s}$ order $\Psi_{0}$ is identified with solution (36) (Fig. 1). The incorporation of dissipation effects has modified the solution of the dynamical non-linear differential equation. It can be seen from Eq. (47) that friction (or collision) is responsible for expo-

10 nential decay of stationary nonlinear vortex structures in space. This street of vortices can be studied by plotting the stream line function $\Psi(\eta, y)$ (Eqs. 47 and 36). We have free parameters $\Psi_{0}^{0}, \kappa$ and $æ_{0}$, and the velocity of movement of the structures $U$ will be determine by (29).

Figure 2 a shows the $k=0.5$ case and $\Psi_{0}^{0}=1, U=0.1, æ_{0}=0.2$, while Fig. $2 c$ shows

15 the $k=1$ case. Three dimensional plots for the same parameters are shown in Fig. 2b and $d$. At decrease of the linear scales of the vortices (with increase of $k$ ) the number of the vortices will increase in the given area of the medium and their amplitudes will decrease (Fig. 2c and d). The reduction in $k$ causes a reduction in number of vortices, e.g., the $k=1$ stream function plots six vortices (Fig. 2c and d). We, therefore, note

20 that the number of vortices increases with increasing $\kappa$, e.g. the formation of nonlinear structures is attributed to low frequency mode.

At decrease of the linear scale of the background wind inhomogeneity (increasing $æ_{0}$ ) the linear scales, amplitudes and steepness of peaks of the vortices decrease accordingly (Fig. 3).

25 The street of vortices is in almost stationary frame of reference, it disappears for higher frame velocity $(U>1)$, i.e. the contribution of logarithmic and hyperbolic trigonometric functions are no longer overcome by the contribution of linear term viz. $U y$ in 1449

Eq. (36) and, therefore, vortex formation is replaced by straight stream lines (Fig. 4). Due to increase of the translation velocity $(U)$ of the structures and the background flows the scales and amplitudes of the generated vortices will decrease. In case of comparably high velocity background wind $(U>1)$ the vortex will not be generated at 5 all and only the background flow will remain in the medium (Fig. 5). Further, due to the nonlinear term, the velocity of dispersive waves must be greater than the phase velocity of a wave which resulted in a bending of the wave front and hence vortices start to form.

The street of vortex disappears in the space for high dissipation rate $\Lambda$ (or collision 10 frequency) (Fig. 6). We credence that the dissipation effect has not permitted the vortex formation, but the topography of stream line function has been modified (Fig. 6).

\section{Relaxation of the vortex structures in the ionosphere}

The real mechanism of dissipation in the atmosphere against the background of baroclinic, nonlinear and dispersive effects generates in the ionosphere moving spatial 15 structures representing the equilibrium stationary solutions (35) and (36) of the governing magneto-hydrodynamic Eqs. (1) and (2). For qualitative estimation of the evolution and the temporal relaxation of stationary vortex structures in the ionosphere, built in previous paragraphs, the dynamic Eqs. (1) and (2) can be approximately written as the following Helmholtz's vortex transfer equation:

${ }_{20} \frac{\partial}{\partial t} \nabla \times \mathbf{V}=\mathbf{P}-\Lambda(\nabla \times V)$

which describes the generation of nonzero vorticity $\nabla \times \mathbf{V}\left((\nabla \times \mathbf{V})_{z}=\Delta \Psi\right)$ in the ionosphere under the action baroclinic vector $\mathbf{P}$ (source function) taking in to account the temperature contrasts in the form of advection of warm and cold, medium dispersion

25 and influence of small nonlinearity. According to the observations (Gill, 1982; Pedlosky, 1987), vector $\mathbf{P}$ for low-frequency disturbances is a slowly varying function of time. In 
this case the vortex Eq. (29), with the initial conditions of Cauchy $\nabla \times\left.\mathbf{V}\right|_{t=0}=0$ (at the initial moment in the atmosphere there no vortices) has the bounded solution:

$\nabla \times \mathbf{V}=\frac{\mathbf{P}}{\Lambda} 1-\mathbf{e}^{-\Lambda t}$

5 Dissipative effects have an accumulative nature and its action becomes perceptible only after a certain interval. From Eq. (49) it follow that vorticity will increase linearly with time only at small time intervals $(t \ll 1 / \Lambda)$ under the action of baroclinicity and some other effects. After a certain time, when the dissipation effect reaches a specific value, vortex growth speed decreases (the vorticity growth rate decreases) and for the 10 large intervals of time $(t \gg 1 / \Lambda)$ it tends to constant (equilibrium) value $\mathbf{P} / \wedge$ (Fig. 7 ). The value of dimensional time $T=1 / \Lambda \approx 10^{5} \mathrm{~s} \geq 24 \mathrm{~h}$ can be called a relaxation time of non-stationary vortex street. Indeed, for the lower atmosphere relaxation time is of the order of twenty-four hours (Gossard and Hooke, 1975; Pedlosky, 1982) and consequently here large scale vortices must be long-lived. Stationary solution describes the

15 equilibrium between baroclinicity and the dissipation effects $(\mathbf{P}=\Lambda(\nabla \times \mathbf{V}))$. As a result, the dissipative structure is generated in the ionosphere in the form of Stationary Street of cyclones and anticyclones.

\section{Conclusions}

Based on the nonlinear dynamical equations, considered in Aburjania et al. (2011), the 20 linear and nonlinear interaction of planetary electromagnetic ultra-low-frequency fast and slow wavy structures with zonal shear flow in the Earth's dissipative ionosphere is investigated. Along with the prevalent effect of Hall conductivity for such waves, the latitudinal inhomogeneity of both the angular velocity of the Earth's rotation and the geomagnetic field becomes essential. Due to spatial inhomogeneity of the Earth's rotation

25 velocity fast and slow waves can be coupled. Such coupling results in an appearance of strong dispersion of these waves. Note that, without this coupling the fast branches 1451

in the both ionospheric $E$ and $F$ regions lose the dispersion property for both large and short wave-length perturbations.

It is show, that at interaction with the inhomogeneous local wind the EM ULF wave perturbations can sufficiently increase own amplitude and energy and in their dynamics

5 the nonlinear effects will be appeared. Dynamical competition of the nonlinear and the dispersion effects at the different layers of the ionosphere creates a favorable condition for self-organization of the EM ULF disturbances into nonlinear vortex structures. The self-localization of the planetary ULF waves into the long-lived solitary vortex streets in the non-dissipative ionosphere is proved in the basis of the analytical solution of 10 the governed nonlinear dynamic equations. The exact stationary solution of these nonlinear equations has an asymptote $\psi \sim \exp (-k r)$ at $r \rightarrow \infty$, so the wave is strongly localized along the Earth surface. The translation velocity $U$ of ULF EM vortices is very crucial which in turn depends on parameters $\beta$ and $\beta_{H}$. From analytical calculation and plots we note that the formation of stationary nonlinear vortex street require some threshold value of translation velocity $U$ (Eq. 29) for both nondissipative and dissipative complex ionospheric plasma. For some large value of the background wind's spreading velocity $(U \geq 10)$ the vortex structures may not be raised at all and only the background wind will be preserved in the medium (Fig. 4). Number of vortices in generated nonlinear structures and a value of amplitudes of these vortices essentially depend on the 20 size of the background wind's inhomogeneity - decreasing the latter - generated vortex's size and amplitude will automatically decrease (Fig. 4). It's shown that the space and time attenuation can not resist the formation of the vortex structures, but affect the topographic features of the structures (Figs. 5 and 6). The generated nonlinear vortex structures are enough long-live $(>24 \mathrm{~h}$ ) in dissipative ionosphere.

25 Depending on the type of velocity profile of the zonal shear flow (wind), the generated nonlinear long-lived vortex structures maybe represent monopole solitary anticyclone or cyclone, the cyclone - anticyclone pair, connected in a certain manner and/or the pure dipole cyclone - anticyclone structure of equal intensity, and/or the vortex street, or the vortex chains, rotating in the opposite direction and moving along the latitudinal 
circles (along the parallels) against a background of the mean zonal wind (see also Jovanovich et al., 2002; Aburjania et al., 2003, 2006, 2007).

The nonlinear large-scale vortices generate the stronger pulses of the geomagnetic field than the corresponding linear waves. Thus, the fast vortices generate the magnetic field $h^{\mathrm{f}} \approx 10^{5} n T$, and the slow vortices form magnetic field $h^{\mathrm{s}} \approx 10^{4} n T$. The formation of such intensive perturbations could be related to the specific properties of the considering low frequency planetary structures. Indeed, they trap the environmental particles, and the charged particles in $E$ and $F$ regions of the ionosphere are completely or partially frozen into the geomagnetic field. That's why, the formation of these structures 10 indicates at the significant densification of the magnetic force lines and, respectively, the intensification of the disturbances of the geomagnetic field in their location. Since, the number of the capture parcels is the order of the passed-by (transient), the perturbation of the magnetic field in the stronger faster vortices would be the same order as of the background field. On the earth surface located $R_{0}\left(\sim(1 \div 3) \times 10^{2} \mathrm{~km}\right)$ below the 15 region of the researching wave structure, the level of the geomagnetic pulses would be less by $\exp \left(-R_{0} / \lambda_{0}\right)$ factor. $\lambda_{0}$ is the characteristic length of the electromagnetic perturbations. Since $\lambda_{0} \sim\left(10 \div 10^{2}\right) R_{0} \gg R_{0}$ the magnetic effect on the earth would be less then in $E$ and $F$ regions, but in spite of this they are easily registered too.

Hence, inhomogeneity of the Earth's rotation along the meridian, geomagnetic field

20 and zonal prevailing flow (wind) can be considered among the real sources generating planetary ULF vortex structures of an electromagnetic nature in the ionosphere.

Author contribution. Statement of the problem, elaboration of the physical model and the governing equations for the ionospheric plasma medium with shear flows belongs to $G$. Aburjania and G. Zimbardo. K. Chargazia and O. Kharshiladze were involved in all stages of problem 25 solving - estimation of the difficulty of the mathematical model and obtaining the analytical solutions in case of the shear flows.

Acknowledgements. The research leading to these results has received funding from the European Union Seventh Framework Programme [FP7/2007-2013] under grant agreement No.

$$
1453
$$

269198 - Geoplasmas (Marie Curie International Research Staff Exchange Scheme) and from grant no 31/14 of Shota Rustaveli National Science Foundation.

\section{References}

Aburjania, G.: Structural turbulences and diffusion of plasmas in the magnetic traps, Plasma Phys. Rep., 16, 70-76, 1990.

Aburjania, G.: Self-organization of acoustic-gravity vortices in the ionosphere before earthquake, Plasma Phys. Rep., 22, 954-959, 1996.

Aburjania, G.: Self-Organization of Nonlinear Vortex Structures and Vortex Turbulence in Dispersive Media, KomKniga, Moscow, 2006 (in Russian).

10 Aburjania, G.: Formation of strong stationary vortex turbulence in the terrestrial magnetosheat, Geomagn. Aeron., 51, 6, 720-729, 2011.

Aburjania, G. and Chargazia, Kh.: Dynamics of the large-scale ULF electromagnetic wave structures in the ionosphere, J. Atmos. Sol.-Terr. Phy., 69, 2428-2441, 2007.

Aburjania, G. and Machabeli, G.: Generation of electromagnetic perturbations by acoustic waves in the ionosphere, J. Geophys. Res. A, 103, 9441-9447, 1998.

Aburjania, G., Khantadze, A., and Kharshiladze, O.: Nonlinear planetary electromagnetic vortex structures in the ionosphere $F$-layer, Plasma Phys. Rep., 28, 586-591, 2002.

Abururjania, G., Jandieri, G., and Khantadze, A.: Self-organization of planetary electromagnetic waves in E-region of the ionosphere, J. Atmos. Sol.-Terr. Phy., 65, 661-671, 2003.

20 Aburjania, G. D., Chargazia, K. Z., Jandieri, G. V., Khantadze, A. G., and Kharshiladze, O. A.: On the new modes of planetary-scale electromagnetic waves in the ionosphere, Ann. Geophys., 22, 1203-1211, doi:10.5194/angeo-22-1203-2004, 2004.

Aburjania, G., Khantadze, A., and Kharshiladze, O.: Mechanism of planetary Rossby wave amplification and transformation in the ionosphere with an inhomogeneous zonal smooth shear wind, J. Geophys. Res., 111, A09304, doi:10.1029/2005JA011567, 2006.

Aburjania, G. D., Chargazia, Kh. Z., Zelenyi, L. M., and Zimbardo, G.: Model of strong stationary vortex turbulence in space plasmas, Nonlin. Processes Geophys., 16, 11-22, doi:10.5194/npg-16-11-2009, 2009.

Al'perovich, L. and Fedorov, E.: Hydromagnetic Waves in the Magnetosphere and the lono-

30 sphere, Springer, Series: Astrophysics and Space Science Library, Vol. 353, ISBN:978-14020-6636-8, 2007. 
Al'perovich, L., Drobgev, V., and Sorokin, V.: On the midlatitude oscillations of thegeomagnetic field and its connection to the dynamical processes in the ionosphere, Geomagn. Aeron., 22, 797-802, 1982.

Bengtsson, L. and Lighthill, J. (Eds.): Intense Atmospheric Vorticesk, Springer-Verlag, Berlin, Heidelberg, 1982.

Burmaka, V. and Chernogor, L.: Clustered-instrument studies of ionospheric wave disturbances accompanying rocket launches against the background of non-stationary natural processes, Geomagn. Aeron., 44, 3, 518-534, 2004.

Cavalieri, D., Deland, R., Poterna, J., Gavin, R.: The correlation of VLF propagation variations with atmospheric planetary-scale waves, J. Atmos. Terr. Phys., 36, 561-574, 1974.

Chagelishvili, G., Rogava, A., and Tsiklauri, D.: The effect of coupling and linear transformation of waves in shear flows, Phys. Rev. E, 53, 6028-6031, 1996.

Cmyrev, V., Marchenco, V., Pokhotelov, O., Stenflo, L., Strel'tsov, A., and Steen, A.: Vortex structures in the ionosphere and magnetosphere of the Earth, Planet Space Sci., 39, 10251030, 1991.

Fagundas, P., Pillat, V., Bolzan, M., Sahai, Y., Becker-Guedes, F., Abalde, J., Aranha, S., and Bittencourt, J.: Observation of F-layer electron density profiles modulated by planetary wave type oscillations in the equatorial ionospheric anomaly region, J. Geophys. Res., 110, A12, doi:10.1029/2005JA011115, 2005.

20 Fairfield, D. H., Otto, A., Mukai, T., Kokubun, S., Lepping, R. P., Steinberg, J. T., Lazarus, A. J., and Yamamoto, T.: Geotail observations of the Kelvin-Helmholtz instability at the equatorial magnetotail boundary for parallel northward fields, J. Geophys. Res., 105, 21159-21173, 2000.

Georgieva, K., Kirov, B., Atanasova, D., and Boneta, A.: Impact of magnetic clouds on the middle atmosphere and geomagnetic disturbances, J. Atmos. Sol.-Terr. Phy., 67, 163-176, 2005.

Gershman, B. N.: Dynamics of the lonospheric Plasma, Nauka, Moscow, 1974 (in Russian).

Gill, E.: Atmosphere-Ocean Dynamic, Academic Press, New York, London, Paris, 1982.

Gossard, E. and Hooke, W.: Waves in Atmosphere, Elsevier, Amsterdam, 1975.

30 Hajkowicz, L. A.: Global onset and propagation of large-scale traveling ionospheric disturbances as a result of the great storm of 13 March 1989, Planet Space Sci., 10, 583-593, 1991.

1455

Hasegawa, A. and Sato, T.: Generation of field aligned current during substorm, in: Dynamics of the Magnetosphere, edited by: Akasofu, S. I. and Reidel, D., Springer, Dordrecht, Netherlands, 529-542, 1979.

Hasegawa, H., Fujimoto, M., Phan, T. D., Reme, H., Balogh, A., Dunlop, M. W., Hashimoto, C., and TanDokoro, R.: Transport of solar wind into Earth's magnetosphere through rolled-up Kelvin-Helmholtz vortices, Nature, 430, 755-758, 2004.

Hones, E. W.: Vortices in magnetospheric plasma flow, Geophys. Res. Lett., 5, 1059-1062, 1978.

Hones, E. W.: Further determination of the characteristics of magnetospheric plasma vortices with ISEE 1 and 2, J. Geophys. Res., 86, 814-819, 1981.

Hones, E. W.: New observations of plasma vortices and insights into their interpretation, Geophys. Res. Lett., 10, 674-677, 1983.

Jovanovich, D., Stenflo, L., and Shukla, P. K.: Acoustic-gravity nonlinear structures, Nonlin. Proc. Geophys., 9, 333-339, 2002.

15 Kamenkovich, V. M. and Monin, A. S. (Eds.): Ocean Physics, Vol. 2: Ocean Hydrodynamics, Nauka, Moscow, 1978.

Kamide, Y. and Chian, C. L.: Handbook of the Solar-Terrestrial Environment, Springer-Verlag, Berlin, Heidelberg, 2007.

Keika, K., Nakamura, R., Volwerk, M., Angelopoulos, V., Baumjohann, W., Retinò, A., Fujimoto, M., Bonnell, J. W., Singer, H. J., Auster, H. U., McFadden, J. P., Larson, D., and Mann, I.: Observations of plasma vortices in the vicinity of flow-braking: a case study, Ann. Geophys., 27, 3009-3017, doi:10.5194/angeo-27-3009-2009, 2009.

Kelley, M. C.: The Earth's Ionosphere, Plasma Physics and Electrodynamics, Academic Press Inc., San Diego, California, 1989.

25 Keiling, A., Angelopoulos, V., Runov, A., Weygand, J., Apatenkov, S. V., Mende, S., McFadden, J., Larson, D., Amm, O., Glassmeier, K.-H., and Auster, H. U.: Substorm current wedge driven by plasma flow vortices: THEMIS observations, J. Geophys. Res., 114, A00C22, doi:10.1029/2009JA014114, 2009.

Kopitenko, Y. A., Komarovskikh, M. I., Voronov, I. M., and Kopitenko, E. A.: Connection between

30 ULF electromagnetic litospheric emission and extraordinary behavior of biological system before the earthquake, Biofizika+, 40, 1114-1119, 1995 (in Russian).

Magnus, K.: Schwingungen, Teubner, Stuttgart, 1976. 
Mallier, R. and Maslowe, S. A.: A row of counter-rotating vortices, Phys. Fluids., 5, 1074-1075, 1993.

Manson, A. H., Heek, C. H., and Gregory, J. B.: Winds and waves (10 min-30day) in the mesosphere and lower thermosphere at Saskatoon, J. Geophys. Res., 86, 9615-9625, 1981.

5 Mikhailovskii, A. B.: Theory of Plasma Instabilities, Vol. 2: Instabilities of an Inhomogeneous Plasma, Atomizdat, Moscow, 1971, Consultants Bureau, New York, 1974.

Monin, A. S. and laglom, A. N.: Statistical Hydrodynamics V 2, Nauka, Moscow, 1967 (in Russian).

Nezlin, M. V.: Rossby solitary vortices on giant planets and in the laboratory, Chaos, 4, 187202, 1994

Otto, A. and Fairfield, D. H.: Kelvin-Helmholtz instability at the magnetotail boundary: MHD simulation and comparison with Geotail observations, J. Geophys. Res., 105, 21175-21190, 2000.

Pedlosky, J.: Geophysical Fluid Dynamics, Springer-Verlag, New York, 1987.

15 Petviashvili, V. I. and Pokhotelov, O. A.: Solitary Waves in Plasma and in the Atmosphere, Philadelphia, PA, USA: Gordon and Breach Science Publishers, 1992.

Pokhotelov, O. A., Parrot, M., Fedorov, E. N., Pilipenko, V. A., Surkov, V. V., and Gladychev, V. A.: Response of the ionosphere to natural and man-made acoustic sources, Ann. Geophys., 13, 1197-1210, doi:10.1007/s00585-995-1197-2, 1995.

20 Reddy, S. C., Schmid, P. J., and Hennigston, D. S.: Pseudospectra of the Orr-Sommerfeld operator, SIAM J. Appl. Math., 53, 15-23, 1993.

Shaefer, L. D., Rock, D. R., and Lewis, J. P.: Detection of Explosive Events by Monitoring Acousticaly-Induced Geomagnetic Perturbations, Lawrence Livermore Laboratory, CA USA, 94550, Livermore, 1999

25 Shakina, N. P.: Dynamics of Atmospheric Fronts and Cyclones, Gidrometizdat, Leningrad, 1985 (in Russian).

Sharadze, Z., Mosashvili, N., Pushkova, G., and Yudovich, L.: Long-period wave disturbances in $E$-region of the ionosphere, Geomagn. Aeron., 29, 6, 1032-1034, 1989.

Snekvik, K., Haaland, S., Østgaard, N., Hasegawa, H., Nakamura, R., Takada, T., Juusola, L., Amm, O., Pitout, F., Rème, H., Klecker, B., and Lucek, E. A.: Cluster observations of a field aligned current at the dawn flank of a bursty bulk flow, Ann. Geophys., 25, 1405-1415, doi:10.5194/angeo-25-1405-2007, 2007.

1457

Sorokin, V. M.: Wave processes in the ionosphere associated with geomagnetic field, Izv. Vuz. Radiofiz+, 31, 10, 1169-1179, 1988 (in Russian).

Stepanyants, Y. A. and Fabrikant, A. L.: Features of the Cherenkov emisión of drift waves in hydrodynamics and in plasma, Sov. Phys. JETP-USSR, 102, 1512-1523, 1992.

5 Timofeev, A. V.: Resonance Phenomena in Plasma Oscillations, Fizmatlit, Moscow, 2000 (in Russian).

Trefenthen, L. N., Trefenthen, A. E., Reddy, S. C., and Driscoll, T. A.: Hydrodynamic stability without eigenvalues, Science, 261, 578-584, 1993.

Vasyliunas, V. M.: Fundaments of current description, in: Magnetospheric Currents, Geophys

10 Monogr. Ser., 28, edited by: Potemra, T. A., AGU, Washington, DC, 63-66, 1984.

Williams, G. P. and Yamagata, T.: Geostrophic regimes, intermediate solitary vortices and Jovian eddies, J. Atmos. Sci., 41, 453-478, 1984.

Whitham, G. B.: Linear and Nonlinear Waves, Wiley, New York, 1974, Mir, Moscow, 1977.

Zel'dovich, Ya. B., and Mishkis, A. D.: Elements of Applied Mathematics, Nauka, Moscow, 1972 (in Russian).

Zong, Q. G.,Wang, Y. F., Yang, B., Zhang, H., Tian, A. M., Dunlop, M., Fritz, T. A., Kistler, L. M., Korth, A., Daly, P. W., and Pedersen, A.: Vortex-like plasma flow structures observed by cluster at the boundary of the outer radiation belt and ring current: a link between the inner and outer magnetosphere, J. Geophys. Res., 114, A10211, doi:10.1029/2009JA014388, 2009.

20 Zhou, Q. H., Sulzer, M. P., and Tepley, C. A.: An analysis of tidal and planetary waves in the neutral winds and temperature observed at low-latitude $E$-region heights, J. Geophys. Res., 102, 491-505, 1997. 


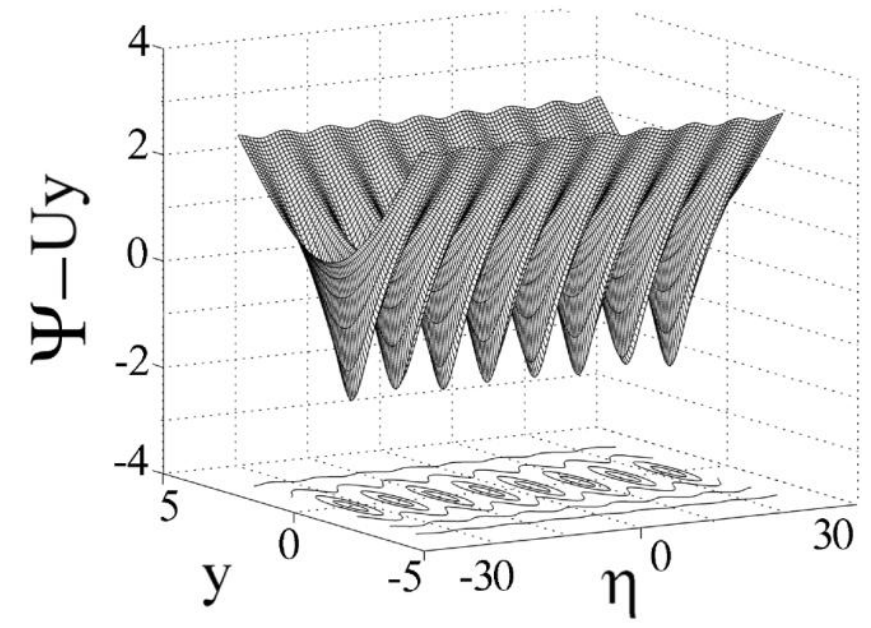

Figure 1. Relief and level lines in the rest frame of the vortices $\Psi(\eta, y)-U y$, calculated from Eq. (55) for $\psi_{0}^{0}=1, k=1, æ_{0}=0.5$ (the longitudinal vortex street).

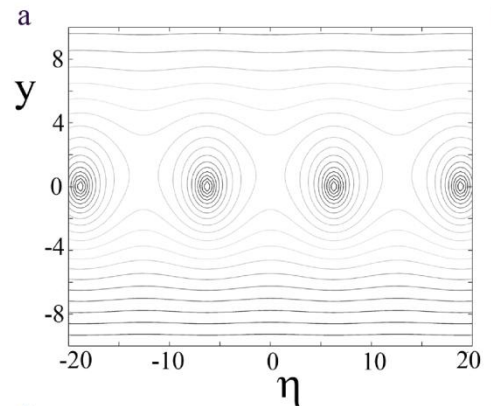

\section{b}
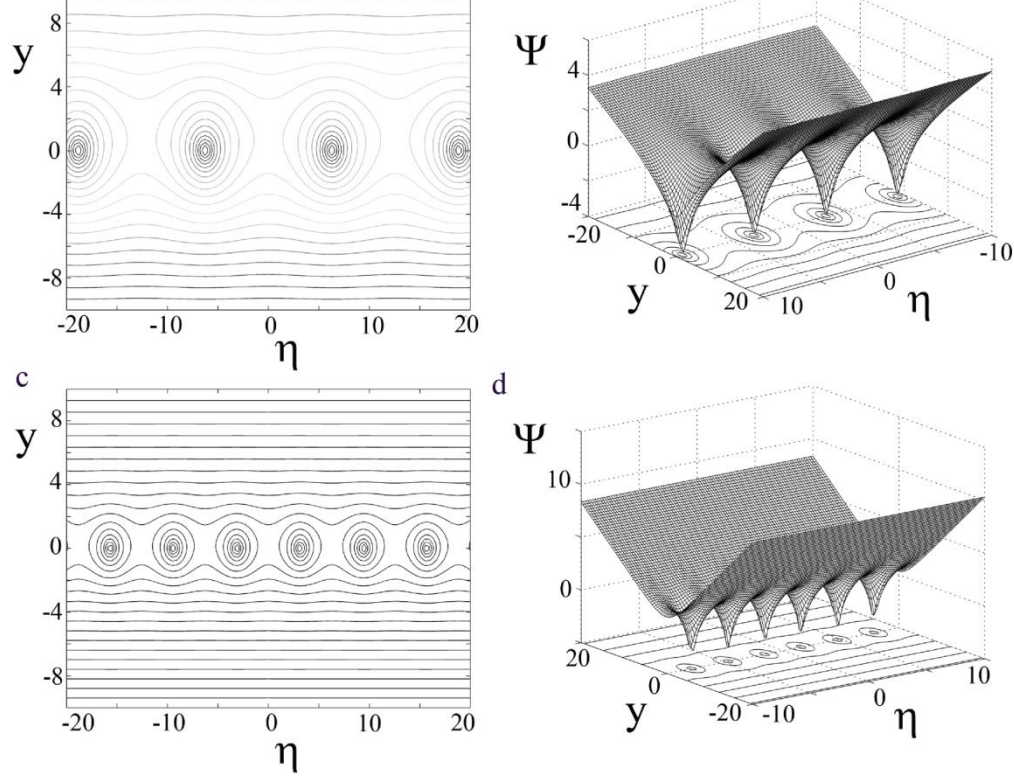

Figure 2. The level lines and relief of the stream function of vortex solution (36) in the moving system of coordinates to the parameters: (a) $\psi_{0}^{0}=1, U=0.1, æ_{0}=0.2, k=0.5$; (b) $\psi_{0}^{0}=1$, $U=0.1, æ_{0}=0.2, k=1$; (c) $\psi_{0}^{0}=1, U=0.1, æ_{0}=0.2, k=0.5$; (d) $\psi_{0}^{0}=1, U=0.1, æ_{0}=0.2$, $k=1$. 

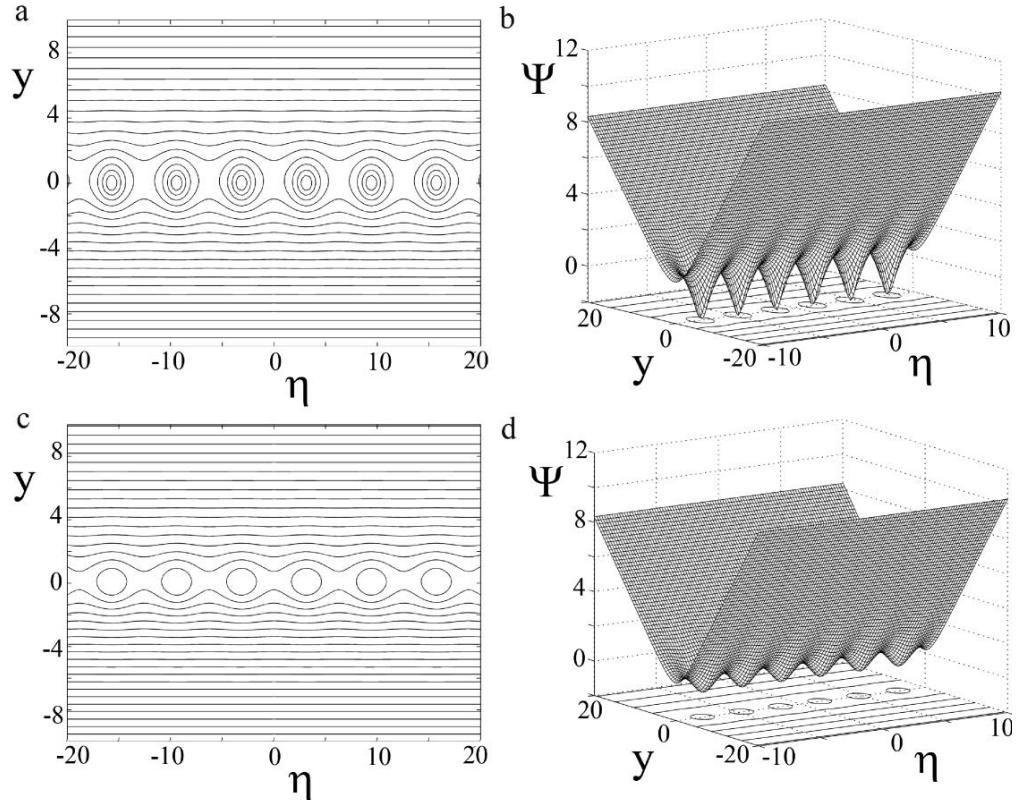

Figure 3. The level lines and relief of the stream function of vortex solution (36) in the moving system of coordinates to the parameters: (a) $\psi_{0}^{0}=1, U=0.1, æ_{0}=0.5, k=1$; (b) $\psi_{0}^{0}=1, U=$ $0.1, æ_{0}=0.5, k=1$; (c) $\psi_{0}^{0}=1, U=0.1, æ_{0}=0.9, k=1 ;$ (d) $\psi_{0}^{0}=1, U=0.1, æ_{0}=0.9, k=1$.
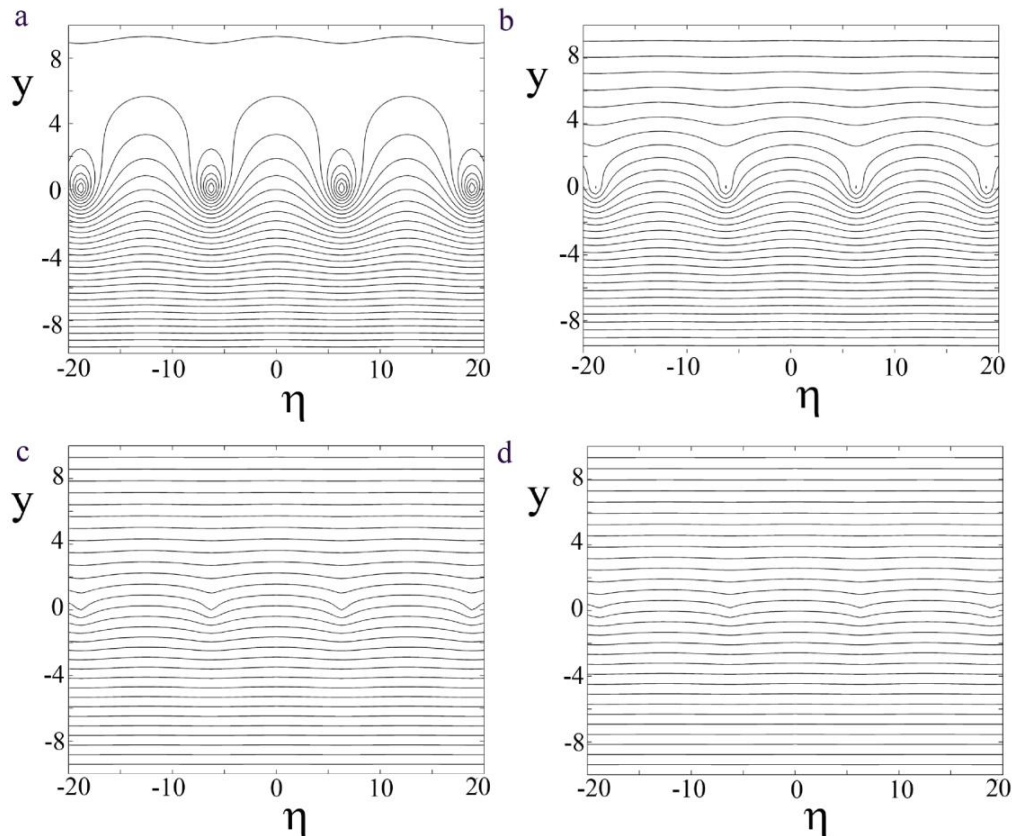

Figure 4. The level lines of the stream function of vortex solution (36) in the moving system of coordinates to the parameters: (a) $\psi_{0}^{0}=1, U=0.6, \mathfrak{x}_{0}=0.2, k=0.5$; (b) $\psi_{0}^{0}=1, U=1.5$, $æ_{0}=0.2, k=0.5$; (c) $\psi_{0}^{0}=1, U=5, æ_{0}=0.2, k=0.5$; (d) $\psi_{0}^{0}=1, U=10, æ_{0}=0.2, k=0.5$. 
a

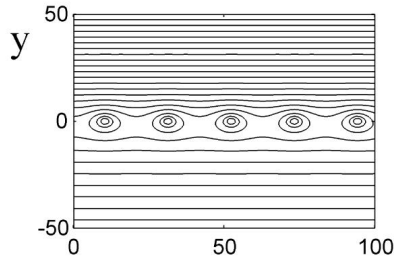

b

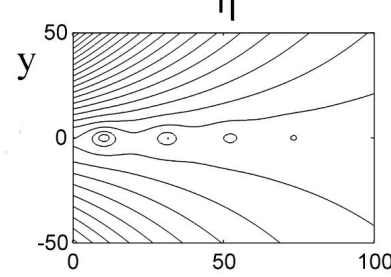

C

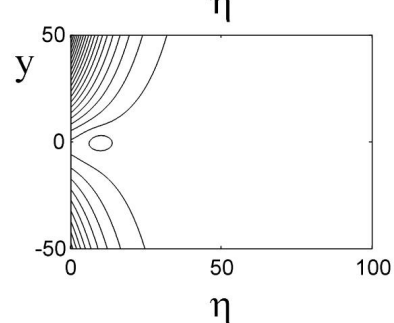

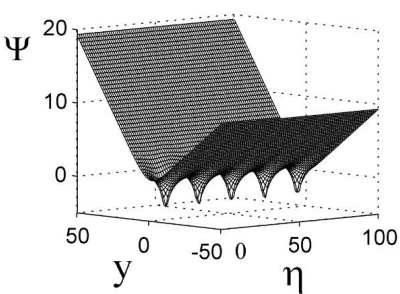
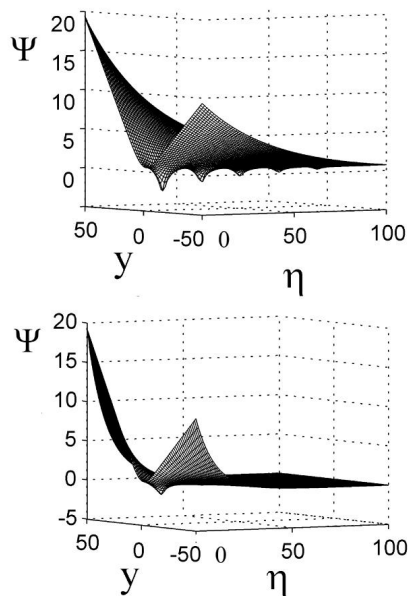

Figure 5. Spatial damping of the vortex structures (the level lines and relief of the stream function), calculated from Eq. (66) to the parameters: (a) $\psi_{0}^{0}=1, U=0.1, æ_{0}=0.2, k=0.3$, $\Lambda=0.000$; (b) $\psi_{0}^{0}=1, U=0.1, æ_{0}=0.2, k=0.3, \Lambda=0.0025$; (c) $\psi_{0}^{0}=1, U=0.1, æ_{0}=0.2$, $\kappa=0.3, \wedge=0.01$.

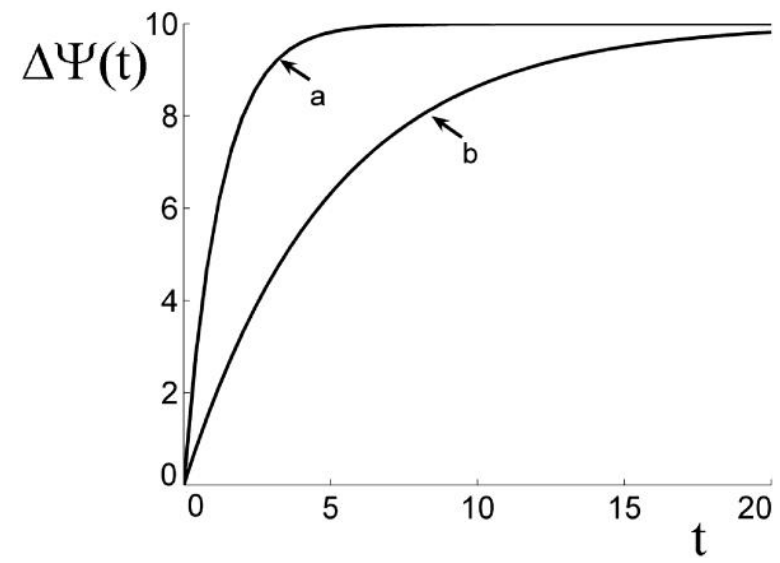

Figure 6. Relaxation of the vorticity of perturbations, calculated from Eq. (49) to the parameters: (a) $\Lambda=0.8, P / \wedge=10 ;$ (b) $\Lambda=0.2, P / \Lambda=10$. 